\title{
Nanotechnology: Next generation war against cancer
}

\begin{tabular}{l} 
Asad Riaz ${ }^{1}$, Aqsa Tahir ${ }^{1}$, Sohail Ahmad Jan ${ }^{2,6 *}$, Nazir Ahmad ${ }^{3}$, Iqra \\
Khalid $^{1}$, Farah Kanwal \\
Khan $^{6}$, Rimsha Zainab ${ }^{7}$ and Haris Khurshid \\
1. Center of Agricultural Biochemistry and Biotechnology, University of Agriculture, Faisalabad-Pakistan \\
2. Department of Biotechnology, Quaid-i-Azam, University, Islamabad-Pakistan \\
3. National Institute for Genomics and Advanced Biotechnology, National Agricultural Research Centre (NARC), \\
Islamabad-Pakistan \\
4. Department of Animal Sciences, Quaid-i-Azam University, Islamabad-Pakistan \\
5. Department of Agricultural Sciences, Allama Iqbal Open University, Islamabad-Pakistan \\
6. Plant Genetic Resources Institute (PGRI), NARC, Islamabad-Pakistan \\
7. Department of Botany, Hazara University, Manshera-Pakistan \\
8. Oilseeds Research Program, NARC, Islamabad-Pakistan \\
*Corresponding author's email: sjan.parc@gmail.com; sohailahmadjan3@gmail.com \\
Citation \\
Asad Riaz, Aqsa Tahir, Sohail Ahmad Jan, Nazir Ahmad, Iqra Khalid, Farah Kanwal, Azka Anwar, Sabir Hussain \\
Shah, Ashtar Khan, Rimsha Zainab and Haris Khurshid. Nanotechnology: Next generation war against cancer. Pure \\
and Applied Biology. Vol. 6, Issue 1, pp352-362. http://dx.doi.org/10.19045/bspab.2017.60034 \\
\hline \hline
\end{tabular} \begin{tabular}{llll}
\hline \hline Received: 21/11/2016 $\quad$ Revised: 23/02/2017 Accepted: 27/02/2017 Online First: & \\
& &
\end{tabular} $02 / 03 / 2017$

\section{Abstract}

The applications of nanotechnology significantly benefit clinical practices in cancer diagnosis, management and treatment. This article reveals the application of nanotechnology in cancer treatment by using the different strategies for example use of nanoparticles like liposomes, dendrimers, nanoshells, carbon nanotubes, superpara-magnetic, silver/gold nanoparticles and nucleic acid based nanoparticles as well as the nanotechnology for a combination of therapeutic strategies. The advantages and challenges of these particles are also discussed. The use of different green synthesized based gold and silver nanoparticles are important for the apoptosis of different types of cancerous cells. Both size and shapes of these particles are important from the biological point of view and from the material characteristics. In present review we have also highlighted the role of some advanced nano-technologies and bio-therapeutics for diagnose and treatment of this lethal disease.

Keywords: Liposomes; Gold nanoparticles; Silver nanoparticles; Super paramagnetic; Antitumor; Bio-therapeutics

\section{Introduction}

Cancer issues and various applications of nanotechnology for cancer therapy

The anti-cancerous drugs which we used are highly targeted and minimum effect on healthy tissues. Nanoparticles which are used in anti-cancer drugs are based on size, surface charge and the ability to recognize the cancerous cells [1]. Research based on multi-functional nanoparticles has quickly been developed against different sort of tumors, its imaging and therapy as well. This system is quick and efficient to control lethal cancer disease. Biodegradable and 
biocompatible polymers are used to prepare nanoparticles for tumor-targeted delivery [2]. About $1 / 8^{\text {th }}$ death occurs in this globe is due to cancer disease. The findings of an international agency research showed that about 13.1 million deaths associated with different types of cancer by 2030. The main reason behind this fact is due to lack of proper naturally or artificially anti-cancer drug producing and delivery systems. Therefore, it is important to develop a new, quick and efficient target delivery system, which can target specific tumor with maximum accuracy without any side effect [3]. Nanoparticles attract great attention due to their small size. Nanoparticles have the ability to move in the tumor tissue through the pores of some health tissue. In other hand the tumor tissue has weak lymphatic circulation system that leads tissue homeostasis and thus increases the retention of these particles. In this case this process is called enhanced permeation and retention effect. In this process, the particular size of drug and its delivery system is very important [4]. Lot of smart polymers which are stimuli-responsive polymers can be activated by $\mathrm{pH}$, temperature, light, electric field or some need dual stimuli like ph and ionic strength. Gold nanoparticles used for cancer treatment. Different subtypes of gold nanoparticles, such as rod shapes, nano cages and nano shell [5]. The advancement of gold nanoparticles for example control of size or refined coating was introduced in 20 century with the immerging of new techniques such as transmission electron microscope. GNPs also have the multifunctional properties to use in cancer treatment and diagnosis such as micellar hybrid nanoparticles containing metal properties for MRI detection. GNPs show good result for cancer treatment. Efficiency has been observed in mouse which implanted human breast cancerous cells. Non-specific receptor mediated endocytosis
(RME) mode used for the entry of GNPs. Enhanced permeability and retention effects observed, nanoparticles passively accumulates at tumor site. PEGlytion is done to achieve the combined effect of RME with longer concentration time, 10-100 fold penetration concentration of drugs will be increased as compared to free drug. Toxicity effects of GNPs have been conflicted, in some studies no toxicity effect were observed at inter or intracellular level. While at cellular level some toxicity were observed. Zinc oxide nanoparticles are very effective to kill the three types of cancerous cells, 1- HepG2 2-A459 and 3-BEAS B2. These were shown some toxic effects but due to antibacterial property these particles were widely used to kill cancerous cells. Toxic effects were observed in neuroblastoma and T-cells $[4,6]$. Several different types of secondary metabolites were used to cure many lethal diseases [7, 8]. However the following new nanoparticles have higher potential to diagnose and treat many lethal diseases including cancer in quick and efficient ways.

\section{Nanoparticle brands Liposomes}

Liposomes are spherical vesicles have a lipid bilayer which encapsulates an aqueous phase to store drug. Due to its hydrophobic interaction liposomes perform excellent delivery of hydrophobic and hydrophilic drugs. Many lipids have different fatty acid chain lengths, different head groups and different melting temperatures. The main advantage of the liposome is fatty layer because it limits and gives fully protection to inserted drugs until it reached to particular adhered tissue. The other advantage of this system is it provided less toxicity to healthy cells as compared to target cancerous cell, where its effect increased many folds. In this manner by using liposomes drug toxicity to healthy cells is decreased and its efficiency for 
cancerous cell may be increased it is a good technology for delivery of chemotherapy drugs. Liposomes encapsulating different therapeutic agents are used as potential nanocarrier for cancer therapy [9].

\section{Dendrimers}

The dendrimers are the highly branched synthesized macro-particles, in which treelike structures are mono-dispersed and three-dimensional. They have well defined molecular weights and have the ability to provide maximum benefit to host. Branches are considered as a useful character of dendrimers which provide a large amount of surface area for drugs and targeting molecules [1, 10]. Due to their cylindrical structure these nano-objects have gained much popularity because these structures provides elite and unique properties which could be utilized in the new advanced field of nanotechnology for the treatment of different types of lethal cancer diseases [10, 11]. For example DNA polyamidoamine dendrimer complex is used due to its high transfection efficiency and very low toxicity for DNA delivery to cell nucleus. Synthesis and functional evaluation of DNA assembled polyamidoamine dendrimer clusters for cancer cell-specific targeting. By conjugation of folate and fluorescein moieties on the surface of a dendrimer is shown to yield molecules which have the ability of targeting to tumor cells through folate receptors. Poly amidoaminedendrimers use as the backbone of multitasking therapeutics are a favorite candidate due to its better solubility in water, lack of auto-immunity response and low poly dispersity [11, 12].

\section{Carbon nanotubes}

A distinct molecular form of carbon atoms which bond with each other and present hexagonal arrangements were made [13]. Carbon nanotubes may be single or multiple walled. In the nanotechnology platforms, carbon nanotubes provide a novel tool for anticancer drug delivery [14]. Besides those carbon nanotubes has the ability to immobilize molecules such as antibodies, DNA and drugs [15]. In nanotechnology we explore our interesting mechanical, optical, electrical and structural properties of singlewalled carbon nanotubes for biological applications including new therapies, biosensors and molecular transporter for drug delivery Cancer nanotechnology: opportunities and challenges. Single walled nanotubes has high optical absorbance near infrared-regime causes heating when there is laser radiation that is useful for destroying cancerous cells. For bio-imaging purpose surface-functionalized carbon nanotubes have also been used [16]. Recently the biological impacts for example cytotoxicity, inflammation, DNA damage induced by multi and single-walled carbon nanotubes have been studied. Carbon nanotubes are nano-devices use for biomarker detection [17]. Kamet al. [18] have found that singlewalled containing carbon nanotubes of 700$1100 \mathrm{~nm}$ are carriers and molecular transporter with very high optical absorbance, which is near-infrared [NIR] light where biological systems are transparent. In this special continuous NIR radiation, because of excessive local heating can cause cell death. In this way, the carrying capability of carbon nanotubes and their intrinsic optical properties can help for the synthesizing new class of important nano-materials against cancer disease

\section{Different gold nanoparticles}

Gold nanoparticles are one of the best options used for cancerous cell detection because they have valuable attributes of light emission and absorption. It has been expected that these attributes of gold nanoparticles can be employed for cancer diagnosis Recently a new technique has been developing in which nanoparticles are coated by the material which has properties of attraction to specific cells or molecules 
e.g. specialized antibodies are attached to nanoparticles of gold which are then attracted to cancerous cells when mixed with containing cancer cells. Then using microscopy applications the mixed sample is examined under a white light. Each type of cancerous cells contains unique protein depending upon which nanoparticles attached to them. The images will show specific patterns light from which location and type of cancer can be determined [19].

Cancer diagnosis and treatment through different types of nanoparticles

Different methods used for the treatment and diagnosis such as chemotherapy and radiation therapy. But these methods show the toxic effects, kill both cancerous and healthy cells. Some other methods such as quantum dots use for diagnosis of cancerous cells but this method also limited. Due to exposure of extended light, they will burn out. On the other hand gold nanoparticles not burnt. Two methods were investigated using gold nanoparticles which kill only cancerous cells, not healthy cells. In first method gold nanoparticles are mostly triangular shaped stick to cancerous by exposure of light near to infrared. In the second method, gold nanoparticles are synthesized by chemotherapy used. Rod shapes gold nanoparticles with a small dose of chemotherapy subjected to cancer cells. Fabricated gold sphere nanoparticles show more cell penetration [20]. Cancer treatment can only be done after its detection. Nanoparticles have tremendous applications for detection or diagnosis of cancerous cells.

\section{Quantum dots}

Quantum dots is one of the important method for diagnose of cancer with the help of illumination. But this method is not efficient as it burnt with continuous exposure to light as compare to gold nanoparticles that show resistant to light. Gold nanoparticle light therapy is highly sensitive technique, which enables doctors to use only few chemical markers to obtain accurate information [21].

\section{Magnetic separation, magnetite for drug delivery systems and magnetic materials as hyperthermia generator}

The magnetic separation technique basically uses super magnetic iron oxide nanoparticles [SPIONs] for targeting and magnetic extraction of cellular compounds for analyzing their chemical, optical, and physical properties. This is a very conducive practice of cell separation from blood samples. Magnetic cell separation is highly productive than others like electric and centrifugal cell separation. It has been profitably practiced for specific cell separation in the blood, gram-positive pathogens [22] and protein purification [23]. Magnetite is commonly used as drug delivery system due to its extensive properties mainly because it can be reached to target tissue with the help of a magnetic field. This system was efficiently used as loading of magnetic nanoparticles contain anti-tumoral drugs and transformation of these materials into target infected tissue via circulatory system. Magnetic nanoparticles reached into the tumor sites by generation of maximum magnetic field. Due to targeted delivery, the overall toxicity will be low [24]. The optimum delivery system depends on upon the internal and external factors like osmotic controlled- delivery, enzymes based delivery, optimum $\mathrm{pH}$ based delivery, temperature based delivery, electromagnetic triggered delivery, dual or poly-sensitive delivery etc. [25]. Zhang and Misra [26] prepared a new novel magnetic drug based targeting carrier containing of magnetic nanoparticles engulfed in dextran-g-poly. The developed method is highly $\mathrm{pH}$ sensitive and very useful when the $\mathrm{pH}$ of cancer and blood cells remained 5-5.5 and 7.4 respectively. This is a very quick and efficient delivery system in acidic regions. Due to magnetize the magnetic system 
accumulated at the desired tissue and caused local hyperthermia. In other conditions require high temperature but designed magnetic system has a lower critical solution temperature of $38^{\circ} \mathrm{C}$ which make it an excellent system for many humans cancer treatment. Magnetic materials are made by combining the useful properties of organic or inorganic components and magnetic nanoparticles. The commonly used polymers are dextran, chitin, collagen and inorganic oxides like $\mathrm{Zno}, \mathrm{Sio}_{2}, \mathrm{Tio}_{2}$ and titanates [27].

Magnetic nanoparticles play important role in diagnosis of many different types of cancer in-vivo. Magnetic nanoparticles work as hyperthermia agents and can be used efficiently as target drug delivery system to destroy cancer cells. This system has great potential to penetrate the particles to specific parts an organism, and remained there until problem is finished. The size range is 50$300 \mathrm{~nm}$. This property induces hyperthermia for cancer treatment [28].

\section{Uses of metal nanoparticles}

Metal nanoparticles have strong absorption and light scattering abilities in the Plasmon resonance. Therefore, silver and gold metal nanoparticles are commonly used in DNA hybridization detection, cell imaging, protein interaction and photo-thermal therapy [29]. The most attractiveness in the use of these nanoparticles in cancer disease is due to surface chemistry, appropriate size scale and the unique optical properties. The improvement in tumor detection and treatment occur by optimizing both size and shape or by joining of these nanoparticles with particular biomarkers [30]. For examples silver and gold spherical nanoparticles of different sizes are prepared in 2009 it is noticed that anti-tumoral ability of nanoparticles depends on concentration and size of nanoparticle. The small sized nanoparticles have higher efficiency than larger ones. Higher irradiation treatment leads to high killing ability and their higher concentration leads to lower survival cells [31].Several others methods are used for proper delivery of these nanoparticles into cancer cells for example; direct injection for easily accessible tumors; intra-operative application for deep tumors and intravasation of metal nanoparticles for inaccessible tumors.

\section{Chemotherapeutics}

PEG based silver and gold nanoparticles act as a transporter of anticancer chemotherapeutics. The advantages of PEG coating are lower agglomeration tendency, high bio-compatibility and lower autoimmune repose. When injected into the body intravenously it retained for maximum time in the solid tumor tissues. After retention in the tumor, NIR irradiation applied and nanoparticles-enhanced selective ablation occurs [32].

\section{Silicate-based materials as vector against tumor cells}

Silicates are promising materials for both medical and industrial fields. Montmorillonite can be loaded with a lot of drugs into its layered structure because it is $\mathrm{pH}$ sensitive and largely used in the biomedical field. Due to its $\mathrm{pH}$ sensitiveness, its characteristics interlayer spacing is determined. This property is used in medicine to deliver active components to neutral and basic media like colon and intestines. This formulation can be used for oral administration of different active components that are not stable in stomach conditions because of the protective role of the silicates; Stomach environment will not destroy them [33].

Gold Nano shells for thermal therapy

Hyperthermia in combination with radiotherapy and chemotherapy is being used in clinical trials to increase the local control of issues; by increasing the apoptotic cell death. Hyperthermia is limited to preclinical treatment. To meet this limitation 
gold nanoshells are also used for the cancer treatment. Metal nanoparticles are used specifically for the treatment of tumor cell and to heat the metal nanoparticles laser beam is used. As a result of this exposure to laser beam electron excitation and relaxation occur in a controlled manner. The diameter of nanoshell is controlled by the diameter of silica and its size is controlled by the amount of gold. Gold nanoshells having 100nm silica core and $15 \mathrm{~nm}$ gold coat are used mostly for research purposes. Gold nanoshell of $100 \mathrm{~nm}$ size maximally accumulated in SR-BR-3 human cancer. A mice treated control group and with gold nanoshell. When laser tuned, $9{ }^{\circ} \mathrm{C}$ temperature of the control group was increased and $37^{\circ} \mathrm{Cof}$ nanoshell with irreversible damage to a tumor cell. Control group does not show good performance by an irreversible death of tumor tissues. Results showed 93\% mice receive gold nanoshell and laser, no affected response alone laser. By using thermo-sensitive coreshell particles as template nanoshell also synthesized [34].

\section{Recent advances in nanotechnology for treatment of lethal cancer disease diagnosis and treatment}

Several advances have been made in nanotechnology from last two decades against different type of cancer diseases (Figure 1, Table 1). The more recent technologies are death of cancerous cells through photonic ablation therapy. The other technologies such as MRI, computed tomography [CT], conjugates between iron oxide nanoparticles with different coating probes and positron-emission tomography [PET] have been developed for clear identification and treatment monitoring of many types of this disease. The otherbio-therapeutics agents such as DNA particles, peptides, miRNA etc. have several advantages than chemotherapeutics drugs as they are smaller in size and cause efficient target tumor cell death. Direct transfer and expression of DNA into disease cells for the therapeutic applications is gene therapy. Mixed nanoparticles were confirmed to be effective for both in vitro and in vivo gene delivery to colon and liver cancer cells through oligo nucleotides RNAi therapies can control the expression of target genes to treat the disease. The replacement therapy and gene knock out system have been advanced by many researchers that retard or completely block the expression of target oncogenes. The protein/peptide therapy leads apoptosis and thus disturbs abnormal cell function [3537]. 
Positron emission tomography (PET)

- Used as nano-probes

- Diagnosis in early stage
Biotherapeutics

- Quick and efficient

Death of tumor cell

- Lock expression of target

oncogene
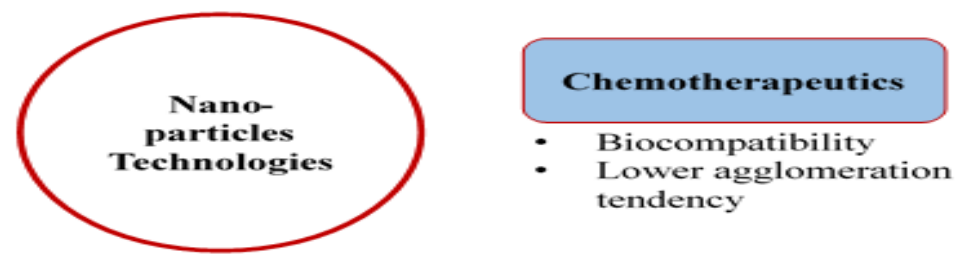

Photonic ablation

therapy

- Disease diagnosis

- Drug delivery system

Figure 1. New technologies used for diagnosis and treatment of cancer disease

Table 1. The applications of Nano based particles against cancer

\begin{tabular}{|l|l|c|}
\hline Nanoparticles & Used against & Reference \\
\hline Gold nanoparticles & HER-2 positive breast cancer & {$[38]$} \\
\hline Doxorubicin-loaded PEG-PLGA nanoparticles & Non-small cell lung cancer & {$[39]$} \\
\hline Gold nanoparticle-hybridized graphene oxide & Breast cancer & {$[40]$} \\
\hline Salinomycin-loaded PLGA nanoparticles & Hepatocellular carcinoma & {$[41]$} \\
\hline Peptide-targeted gold nanoparticles & Brain Cancer & {$[42]$} \\
\hline $\begin{array}{l}\text { CREKA-conjugated dextrancoated iron oxide } \\
\text { nanoparticles }\end{array}$ & Non-small lung cancer & {$[43]$} \\
\hline Liposomes & Glioblastoma multiforme & {$[44]$} \\
\hline Gold nanorods & Prostate cancer & {$[45]$} \\
\hline Cobalt ferrite nanoparticles & $\begin{array}{l}\text { Several types of FR-positive } \\
\text { tumors }\end{array}$ & {$[46]$} \\
\hline $\begin{array}{l}\text { PEI-coated gold NPs with chitosan-aconitic anhydride } \\
\text { and shRNA }\end{array}$ & decrease of ABCG2 expression & {$[47]$} \\
\hline PEG-PEI co-polymer/shRNA & $\begin{array}{l}\text { Effective inhibition of EZH2 } \\
\text { expression }\end{array}$ & {$[48]$} \\
\hline jetPEI-based NPs with CXCR4 shRNA & Melanoma & {$[49]$} \\
\hline Triple-functional pRNA-3WJ nanoparticles & Triple-negative breast cancer & {$[50]$} \\
\hline CD44-targeted shRNA delivered by PLGA-based NPs & $\begin{array}{l}\text { Retard angiogenesis and } \\
\text { apoptosis }\end{array}$ & {$[51]$} \\
\hline $\begin{array}{l}\text { Hydroxyapatite nanoparticles-delivered plasmid based } \\
\text { SATB1 shRNA }\end{array}$ & Human glioma U251 cells & {$[52]$} \\
\hline Silver nanoparticles (AgNPs) & $\begin{array}{l}\text { sub-G1 arrest and apoptosis of } \\
\text { cancerous cell }\end{array}$ & {$[53]$} \\
\hline AgNPs & $\begin{array}{l}\text { Reactive Oxygen Species } \\
\text { mediated cancerous cell damage }\end{array}$ & {$[54]$} \\
\hline Silver -selenium nanoparticles & $\begin{array}{l}\text { Antibacterial and anticancer } \\
\text { activities }\end{array}$ & {$[55]$} \\
\hline
\end{tabular}




\section{Conclusion}

Nanotechnology is of great interest for medical purposes. Different nanoparticles are discussed so far to show their importance and role in cancer diagnosis and treatment. Along with the diagnosis of cancerous cells, targeted delivery of cancer curing drug was also the main focus of this article. Liposomes, carbon nanotube and nanoshells are used to identify and treat the tumor but for highly targeted drug delivery magnetite are used which had wide range applications like hyperthermia therapy, MRI, tissue engineering etc in clinical diagnosis and handlings. Silver and gold nanoparticles are also used for detection and cure. Their appropriate size to shape ratio is desired for their coveted action. The various green synthesized silver and gold nanoparticles lead the apoptosis or cellular damage of cancerous cells. The new advanced technologies have achieved major success for controlling of this lethal disease. In short nanotechnology has huge applications in near future to combat the war against cancer.

\section{Authors' contributions}

Retrieved literature, formatting and manuscript writing: A Riaz, A Tahir, SA Jan, N Ahmed, I Khalid, F Kanwal, A Anwar \& SH Shah, Proofreading, editing, formatting, figure and table preparation: SA Jan, A Khan, R Zainab \& H Khurshid.

\section{References}

1. Ficai D, Andronescu E, Ficai A, Voicu G, Vasile B, Ionita V \& Guran C (2012). Synthesis and characterization of mesoporous magnetite based nanoparticles. Curr Nanosci 8: 875879.

2. Chawla JS \& Amiji MM (2002). Bio degradable poly [epsilon-caprolactone] nanoparticles for tumor-targeted delivery of tamoxifen. Int J Pharm 249: $127-138$.
3. Peppas LB \& Blanchette JO (2004). Nanoparticle and targeted systems for cancer therapy. Adv Drug Del Rev 56: 1649.

4. Tong R \& Cheng JJ (2007).Anticancer polymeric nano-medicines. Polymer Rev 47: 345-381.

5. Ferrari M (2005). Cancer nanotechnology: opportunities and challenges. Nat Rev Cancer 5: 161171.

6. Marques C, Ferreira JMF, Andronescu E, Ficai D, Sonmez M \& Ficai A (2014). Multifunctional materials for bone cancer treatment. Int J Nanomed 9: 2713-2725.

7. Qasim M, Khalid M, Sayyed A, Din I, Hayat K, Roshni R \& Jan SA (2016). Phytochemical potentials and medicinal uses of twenty-four selected medicinal plants from Swabi, Pakistan. J Rural Dev Agri 1(1): 49-58.

8. Jan SA, Shinwari ZK, Zeb A, Khalil AT \& Shah SH (2015). Ethnobotany and research trends in Trachyspermum ammi L. (Ajowan); a popular folklore remedy. American-Eurasian Journal of Agricultural \& Environmental Sciences 15: 68-73.

9. Immordino ML, Dosio F \& Cattel L (2006). Stealth liposomes: review of the basic science, rationale, and clinical applications, existing and potential. Int J Nanomed 1: 297-315

10. Caraceni A, Brunelli C, Martini C, Zecca E \& De Conno F (2005).Cancer pain assessment in clinical trials. $J$ Pain Symp Manage 29: 507-519.

11. Zhang A, Zhang B, Wachtersbach E, Schmidt M \& Schluter AD (2003). Efficient synthesis of high molar mass, first to fourth generation distributed dendronized polymers by the macromonomer approach. Chem Eur J 9: 6083-6092. 
12. Choi Y, Thomas T, Kotlyar A, Islam MT \& Baker JR (2001). Synthesis and functional evaluation of DNAassembled polyamidoamine dendrimer clusters for cancer cell-specific targeting. Chem Biol 12: 3543-3552.

13. Medeiros SF, Santos AM, Fessi H \& Elaissari A (2011).Stimuli-responsive magnetic particles for biomedical applications. Int J Pharma 403: 139161.

14. Bachilo SM, Strano MS, Kittrell C, Hauge RH, Smalley RE \& Weisman RB (2002). Structure-assigned optical spectra of single-walled carbon nanotubes. Science 298(5602): 23612366.

15. Ruiz A, Salas G, Calero M, Hernandez Y, Villanueva A, Herranz F, Veintemillas-Verdaguer S, Martinez E, Barber DF \& Morales MP (2013). Short-chain PEG molecules strongly bound to magnetic nanoparticle for MRI long circulating agents. Acta Biomater 9: 6421-6430.

16. Vlad M, Andronescu E, Grumezescu AM, Ficai A, Voicu G, Bleotu C \& Chifiriuc MC (2014). Carboxymethylcellulose $/ \mathrm{Fe}_{3} \mathrm{O}_{4}$ nanostructures for antimicrobial substances delivery. Biomed Mat Engin 24: 1639-1646.

17. Luo B, Xu SA, Ma WF, Wang WR, Wang SL, Guo J, Yang WL, Hu JH \& Wang CC (2010). Fabrication of magnetite hollow porous nanocrystal shells as a drug carrier for paclitaxel. $J$ Mat Chem 20: 7107-7113.

18. Kam NWS, O'Connell M, Wisdom JA \& Dai H (2001). Carbon nanotubes as multifunctional biological transporters and near-infrared agents for selective cancer cell destruction. PNAS 102: 11600-11605.

19. Mayoral A, Vazquez-Duran A, Barron $\mathrm{H}$ \& Jose-Yacaman M (2009). Polyhedral shaped gold nanoparticles with outstanding near-infrared light absorption. App Phy A: Mat Sci Proc 97: 11-18.

20. Jain S, Bch, MB, Hirst DG \& Sullivan JMO (2012). Gold nanoparticles as novel agents for cancer therapy. $\mathrm{Br} J$ Radiol 85: 101-113.

21. Soppimath KS, Betageri GV \& Cho MH (2008). Nano sctructures for cancer diagnostics and therapy. In $\mathrm{K}$. Gonsalves, C. Halberstadt, C. Laurencin \& L., Nair [Eds.]. Biomedical nanostructures, New York: John Wiley and Sons Inc 409-437.

22. Lin YS, Tsai PJ, Weng MF \& Chen YC (2005). Affinity capture using vancomycin bound magnetic nanoparticles for the MALDI-MS analysis of bacteria. Anal Chem 77: 1753-1760.

23. Franzreb M, Siemann-Herzberg M, Hobley TJ \& Thomas OR (2006). Protein purification using magnetic adsorbent particles. Appl Microbiol Biotechnol 70: 505-516.

24. Kayal S \& Ramanujan RV (2010). Doxorubicin loaded PVA coated iron oxide nanoparticles for targeted drug delivery. Mat Sci Eng C-Mat Biol Appl 30: 484-490.

25. Liang M, Yang TM, Chang HP \& Wang YM (2015). Dual-responsive polymerdrugnano-particles for drug delivery. React Func Poly 86: 27-36.

26. Zhang J \& Misra RDK (2007). Magnetic drug-targeting carrier encapsulated with thermo-sensitive smart polymer: core-shell nanoparticle carrier and drug release response. Acta Biomater 3: 838-850.

27. Jordan A, Scholz R, Wust P, Fahling H \& Felix R (1999). Magnetic, Fluid Hyperthemia [MFH]: cancer treatment with AC magnetic field induced excitation of biocompatible 
superparamagnetic nanoparticles. J Mag Mag Mat 201: 413-499.

28. Khlebtsov NG \& Dykman LA (2010). Optical properties and biomedical applications of plasmonic nanoparticles. J Quant Spect Rad Trans 111: 1-35.

29. Jain PK, El-Sayed IH \& El-Sayed MA (2007). Au nanoparticles target cancer. Nano Today 2: 18-29.

30. Guo SR, Gong JY, Jiang P, Wu M, Lu Y \& Yu SH (2008). Biocompatible, luminescent silver phenol formaldehyde resin core/shell nanospheres: large-scale synthesis and application for in vivo bioimaging. Adv Func Mat 18: 872-879.

31. Xu R, Ma J, Sun XC, Chen ZP, Jiang XL, Guo ZR, Huang L, Li Y, Wang M, Wang CL, Liu JW, Fan X, Gu JY, Chen X, Zhang Y \& Gu N (2009). Ag nanoparticles sensitize IR-induced killing of cancer cells. Cell Res 19: 1031-1034.

32. Khlebtsov NG \& Dykman LA (2010). Optical properties and biomedical applications of plasmonic nanoparticles. J Quant Spect Rad Trans 111: 1-35.

33. Iliescu RI, Andronescu E, Ghitulica CD, Berger D \& Ficai A (2011). Montmorillonite-alginate nanocomposite beads as drug carrier for oral administration of carboplatin preparation and characterization. $U P B$ Sci Bull, Series B: Chem Mat Sci 73: 3-16.

34. Suzuki D \& Kawaguchi H (2005). Gold nanoparticle localization at the core surface by using thermosensitive coreshell particles as a template. Langmuir 21: 12016-12024.

35. Revia RA \& Zhang M (2016). Magnitite nanoparticles for cancer diagnose, treatment, and monitoring: recent advances. Mater Today 19: 157-168.
36. Mornet S, Vasseur S, Grasset F \& Duguet E (2004). Magnetic nanoparticle design for medical diagnosis and therapy. J Mater Chem 14: 2161-2175.

37. Ding JX, He CL, Xiao CS, Chen J, Zhuang XL \& Chen XS (2012). pHresponsive drug delivery systems based on clickable poly [L-glutamic acid]-grafted comb copolymers. Macromol Res 20: 292-301.

38. Lee H, Dam DH, Ha JW, Yue J \& Odom TW (2015). Enhanced human epidermal growth factor receptor 2 degradation in breast cancer cells by lysosome-targeting gold nanoconstructs. ACS Nano 9: 98599867.

39. Alibolandi M, Ramezani M, Abnous K, Sadeghi F, Atyabi F, Asouri M, Ahmadi AA \& Hadizadeh F (2015). In vitro and in vivo evaluation of therapy targeting epithelial-cell adhesionmolecule aptamers for non-small cell lung cancer. J Cont Rele 209: 88-100.

40. Yang L, Tseng YT, Suo G, Chen L, Yu J, Chiu WJ, Huang CC \& Lin $\mathrm{CH}$ (2015). Photothermal therapeutic response of cancer cells to aptamergold nanoparticle-hybridized graphene oxide under NIR illumination. ACS Appl Mater Inter 7: 5097-5106.

41. Jiang J, Chen H, Yu C, Zhang Y, Chen M, Tian S \& Sun C (2015). The promotion of salinomycin delivery to hepatocellular carcinoma cells through EGFR and CD133 aptamers conjugation by PLGA nanoparticles. Nanomed (Lond) 10: 1863-1879.

42. Meyers JD, Cheng Y, Broome AM, Agnes RS, Schluchter MD, Margevicius S, Wang X, Kenney ME, Burda C \& Basilion JP (2015). Peptide-targeted gold nanoparticles for photodynamic therapy of brain cancer. Part Syst Charact 32: 448-457. 
43. Kruse AM, Meenach SA, Anderson KW \& Hilt JZ (2014). Synthesis and characterization of CREKAconjugated iron oxide nanoparticles for hyperthermia applications. Acta Biomater 10: 2622-2629.

44. Madhankumar AB, Slagle-Webb B, Mintz A, Sheehan JM \& Connor JR (2006). Interleukin-13 receptortargeted nanovesicles are a potential therapyfor glioblastoma multiforme. Mol Cancer Ther 5: 3162-3169.

45. Wolfe T, Chatterjee D, Lee J, Grant JD, Bhattarai S, Tailor R, Goodrich G, Nicolucci P \& Krishnan S (2015). Targeted gold nanoparticles enhance sensitization of prostate tumors to megavoltage radiation therapy in vivo. Nanomed 11: 1277-1283.

46. Park BJ, Choi KH, Nam KC, Ali A, Min JE, Son H, Uhm HS, Kim HJ, Jung JS \& Choi EH (2015). Photodynamic anticancer activities of multifunctional cobalt ferrite nanoparticles in various cancer cells. $J$ Biomed Nanotechnol 11: 226-235.

47. Chen Z, Zhang L, He Y, Shen Y \& Li Y (2015). Enhanced shRNA delivery and ABCG2 silencing by charge-reversible layered nanocarriers. Small 11: 952962.

48. Wu Y, Yu J, Liu Y, Yuan L, Yan H, Jing J \& Xu G (2014). Delivery of EZH2shRNA with mPEG-PEI nanoparticles for the treatment of prostate cancer in vitro. Int J Mol Med 33:1563-1569.

49. André ND, Silva VA, Ariza CB, Watanabe MA \& De Lucca FL (2015). In vivo knockdown of CXCR4 using jetPEI/CXCR4 shRNA nanoparticles inhibits the pulmonary metastatic potential of B16-F10 melanoma cells. Mol Med Rep 12: 8320-8326.

50. Shu D, Li H, Shu Y, Xiong G, Carson WE, Haque F, Xu R \& Guo P (2015). Systemic delivery of anti-miRNA for suppression of triple negative breast cancer utilizing RNA nanotechnology. ACS Nano 9: 9731-9740.

51. Shah V, Taratula O, Garbuzenko OB, Taratula OR, Rodriguez-Rodriguez L \& Minko T (2013). Targeted nanomedicine for suppression of CD44 and simultaneous cell death induction in ovarian cancer: an optimal delivery of siRNA and anticancer drug. Clin Cancer Res 19: 6193-6204.

52. Chu SH, Zhou ZM, Feng DF \& Ma YB (2014). Inhibition of human glioma U251 cells growth in vitro and in vivo by hydroxyapatite nanoparticle assisted delivery of short hairpin RNAs against SATB1. Mol Biol Rep 41: 977-9786.

53. Mukherjee S, Chowdhury D \& Kotcherlakota R et al. (2014). Potential theranostics application of biosynthesized silver nanoparticles (4-in-1 system). Theranostics 4(3): 316-335.

54. Chang Y-J, Tai C-J \& Kuo L-J et al. (2011). Glucose-regulated protein 78 (GRP78) mediated the efficacy to curcumin treatment on hepatocellular carcinoma. Ann Surg Oncol 18(8): 2395-2403.

55. Mittal AK, Thanki K, Jain S \& Banerjee UC (2016). Comparative studies of anticancer and antimicrobial potential of bioinspired silver and silverselenium nanoparticles. Appl Nanomedicine 1(1): 1-6. 